\title{
Vigencia del Diccionario Araucano de Félix de Augusta, a cien años de su publicación
}

\author{
Benjamín J. Molineaux \\ Universidad de Edimburgo, Escocia, Reino Unido
}

\begin{abstract}
Resumen
El trabajo que sigue presenta al Diccionario Araucano de Félix de Augusta (1916) en su contexto social y lingüístico original, para luego evaluarlo en el marco de la lengua y sociedad mapuches de cien años más tarde. Se examinan los factores que hicieron del Diccionario un referente excepcional para la difusión y estudio académico del mapudungun, poniéndolos en contrapunto con aquellos factores que conspiran contra su vigencia y utilidad hoy. Evaluamos, además, la dificultad de la actualización o reemplazo de esta obra, justo en el momento histórico en que la lengua más necesita de un referente léxico. Finalizamos con algunos prospectos para la lexicografía mapuche del siglo XXI y para el uso del Diccionario en proyectos de revitalización y lingüística histórica.
\end{abstract}

Palabras clave: mapudungun, Félix de Augusta, lexicografía, neologismos, lingüística histórica.

* Para correspondencia, dirigirse a: Benjamín J. Molineaux (benjamin.molineaux@ed.ac. uk), The Angus McIntosh Centre for Historical Linguistics, School of Philosophy, Psychology and Language Sciences, The University of Edinburgh, Dugald Stewart Building, Room 2.01, 3 Charles Street, Edinburgh, EH8 9AD, Scotland, UK. 


\title{
The validity of Felix de Augusta's Diccionario Araucano, one HUNDRED YEARS AFTER ITS PUBLICATION
}

\begin{abstract}
The following article presents Félix de Augusta's Diccionario Araucano [Araucanian Dictionary] (1916) in its original social and linguistic context, in order to assess its validity in relation to Mapuche language and culture a century later. We examine the factors that made the Dictionary an exceptional reference for the dissemination and academic study of Mapudungun, placing these in counterpoint with factors that conspire against the work's currency and usefulness today. Difficulties are also evaluated with regards to the updating or replacement of this resource at a historical juncture where the language's lexicon is in greatest need of anchoring. The paper concludes with some prospects for $21^{\text {st }}$-century Mapuche lexicography and for the use of the Dictionary in revitalization and historical linguistics projects.

Keywords: Mapudungun, Felix de Augusta, lexicography, neologisms, historical linguistics
\end{abstract}

Recibido: 31/03/16 Aceptado: 06/05/16

El centenario del Diccionario Araucano-Español y Español-Araucano del capuchino Félix José de Augusta debiera ser una ocasión celebratoria. Es, antes que todo, la conmemoración del primer diccionario moderno de la lengua mapuche -una obra contundente, de excepcional sistematicidad, utilidad y cobertura. Sin embargo, la instancia se nos presenta algo más sobria al ver que un siglo entero ha pasado sin producir una sola obra lexicográfica que supere - por cobertura- la de Augusta ${ }^{1}$. ¿Por qué debiera ser superada? De su excelencia no cabe duda, ni de su adecuación a la cultura tradicional mapuche (v. Salas 1985; Gallardo 1986). Aun más, su antigüedad garantiza una menor influencia del español, preservando sus rasgos tipológicos distintivos. Sin embargo, un diccionario - tratándose de una lengua viva- no ha de ser un frágil documento histórico, sino un receptáculo del dinamismo

\footnotetext{
1 En la presente nota, citamos la edición original de 1916 ('Diccionario'), dando los números romanos de página para la Introducción al Tomo I, sin hacer referencia adicional al autor y año. Al referirnos a una entrada particular, la palabra o morfema relevante se da en cursiva.
} 
léxico de una cultura, siendo su naturaleza estar siempre en rezago del vigoroso paso de la usanza lingüística real.

Los cambios del último siglo en el mundo mapuche son innegables, al igual que los cambios en el trabajo académico sobre el mapudungun. ¿Por qué, entonces, no hay cambio en su registro léxico? ¿Es acaso un descuido de las nuevas generaciones de lexicógrafos del mapudungun?, ¿o es simplemente que hay muy poco cambio que documentar, en tanto la lengua pierde hablantes y vitalidad (v. Gundermann et al. 2011)? Hay una tercera opción, sin embargo: que el temor a dejar registro de una avalancha de cambios tales, se perciba como decadencia y derrota de la lengua, y que en su purismo, las nuevas generaciones dejen languidecer el trabajo lexicográfico real en pos de una ficción.

Como veremos, el Diccionario cubre dos tomos (mapudungun>español y español>mapudungun), y más de setecientas tupidas páginas. Comparado con los 'vocabularios' que lo antedatan -compuestos por otros misioneros (Valdivia 1606; Febrés 1765; Havestadt 1777), viajeros (Herckmans 1642-1643; Falkner 1774), académicos (Cañas 1911) y militares (Barbará 1879) - es considerablemente más extenso y presenta ejemplificación mucho más detallada. Además, el trabajo de Augusta supera a sus predecesores (exceptuando a Febrés) en su bidireccionalidad, pues éstos tienden a ir exclusivamente del mapudungun al español (o al latín o inglés en el caso de Havestadt/Herckmans y Falkner, respectivamente). Entre los trabajos lexicográficos que preceden al de Augusta merece una mención honrosa el Diccionario Etimológico de Rodolfo Lenz (1905-1910), el cual, sin versar explícitamente sobre el mapudungun, y siendo un trabajo diacrónico más que sincrónico, sirvió a Augusta en la composición de su propia obra.

Aunque el Diccionario continúa siendo la referencia lexicográfica estándar para el mapundungun, no es la más reciente de su género. Contamos, por ejemplo, con un contundente diccionario mapudungun-inglés anexado a la gramática de Smeets (2008), y con un glosario mapudungun-español bien estructurado, aunque breve, en la gramática de Zúñiga (2006). Existe también un atractivo pero acotado diccionario ilustrado trilingüe de lengua y cultura mapuche (mapudungun, inglés, español) de Hernández et al. (1997) y un diccionario bidireccional un tanto más largo, escrito según los principios grafémicos del profesor Ranguileo ${ }^{2} \mathrm{y}$ compilado por el colectivo transandino Wixaleyiñ (Cañumil et al. 2016).

\footnotetext{
2 Donde no se representa directamente la ortografía de Augusta, usamos aquí el alfabeto académico o unificado - v. Croese et al. (1978). Un resumen del tema de la escritura mapuche se encuentra en Zúñiga (2001).
} 
Por su tamaño y fineza de producción, el Diccionario Comentado de Esteban Erize (1960) pareciera ser el sucesor idóneo para el trabajo de Augusta. Desafortunadamente, la utilidad de esta obra es limitada, tanto por una tendencia a 'comentar' de manera excesivamente libre, descuidando la organización, transcripción y ejemplificación de las entradas, y por no contener material nuevo, siendo una síntesis un tanto idiosincrática de otros trabajos sobre la lengua (v. Olivera 1966). En apariencia más breve y modesto, el trabajo lexicográfico contemporáneo más valioso quizás sea el Diccionario Lingüistico-Etnográfico de María Catrileo (1995). Su abundante ejemplificación (con traducciones al español y al inglés) y su énfasis en la transcripción fonémica de las entradas son particularmente bienvenidos.

Como veremos, aunque algunas de estas obras logran refinamientos importantes en comparación con el Diccionario de Augusta, ninguno de ellos trae consigo aquel perogrullesco elemento con el cual un trabajo nuevo puede superar a uno antiguo: su adecuación a los tiempos. Los mencionados diccionarios y glosarios se circunscriben al ámbito tradicional mapuche y, en tanto, no extienden sus elementos léxicos para abarcar la variada experiencia de ser mapuche en el siglo $\mathrm{XXI}^{3}$.

\section{AUGUSTA Y EL MAPUDUNGUN DE PRINCIPIOS DEL SIGLO XX}

Fueron años de temor, de pestes, de hambre, de pérdida de una identidad y reformulación de una nueva cultura como minoría étnica enclavada en la sociedad rural chilena.

(Bengoa 2000: 329)

Tras su brutal derrota a manos del ejército chileno, los mapuches de fin de siglo XIX se vieron desplazados y, por primera vez, robados de autodeterminación. La Pacificación de la Araucanía (1861-1884) fue, sin

\footnotetext{
3 La única posible excepción es el diccionario de Smeets, basado en el trabajo de campo de su tesis doctoral, realizado en los años 80 . La gran desventaja de este trabajo, además de prestar equivalencias al inglés, es su carácter académico, que requiere un usuario versado en materias lingüísticas y, en particular, en las categorías de la gramática que precede al diccionario.
} 
más, una campaña de anexión territorial que trajo consigo la anexión de un pueblo completo -reticente, pero desarticulado. Aunque los mapuches recibieron terrenos de 'merced', estos eran mayoritariamente pequeños y de peor calidad que aquellos que se otorgaron a colonos o se pusieron a subasta. A menudo, la entrega demoró, o los títulos fueron usurpados por medio de los subterfugios propios de la creciente industria de especulación y latifundismo. Reducidos a un sedentarismo forzado, la forma de vida tradicional de los mapuches dejó de ser practicable. En su lugar, las reducciones -comunidades mapuche compuestas de un pequeño número de familias asociadas a un lonko- se transformaron en islas de cultura y lengua mapuche en medio de un mar de chilenidad rural. El único camino a seguir para el mapuche tornado microagricultor, era -a ojos del Estado y gran parte de los mismos afectados- la integración a la sociedad chilena y su clase proletaria.

La llaga de la derrota y el desplazamiento estaba aún plenamente abierta cuando, en los últimos días de 1895, arribó la primera misión de capuchinos bávaros a la Araucanía. Entre ellos venía el compilador del Diccionario que nos concierne, de unos 35 años y médico de profesión. Nacido en la ciudad alemana de Augsburg, August Stephan Kathan, bajo su nombre eclesiástico castellanizado de Félix José de Augusta, habría de permanecer en territorios mapuche cuatro décadas, hasta su muerte en Valdivia en 1935. La mayor parte de su misión se realizó en el sector de Huapi, provincia de Cautín (La Araucanía), entre la desembocadura del río Imperial y el lago Budi, una zona de autodenominación lafkenche que era entonces -y continúa siendo- de las de mayor proporción poblacional mapuche.

El objetivo de los puntos de avanzada misioneros era primordialmente educacional y evangélico. Sin embargo, la aproximación de los capuchinos era menos fuertemente instrumental que las misiones anglicanas o las escuelas estatales, donde el énfasis era siempre la integración y la negación de la cultura y lengua ancestral (Arellano 2010). Los bávaros mostraron interés por el idioma y las prácticas indígenas, produciendo no solo importantes trabajos lingüísticos (v. Augusta y Moesbach), sino también trabajos etnográficos muy relevantes (como los de Sigfrido Frauenhäusl y Sebastián Englert de Dillingen). En el caso de Augusta, su conocimiento práctico de la lengua mapuche procede tanto de una insólita dedicación a su labor -"llegaba a olvidar las horas de comida y sueño" (Salas 1985: 208)-, como de su contacto cercano con los hablantes- trabajando sobre temas lingüísticos con sus informantes "velaban ... hasta horas avanzadas de la noche" (iv-v). No cabe duda, además, de que su vocación pedagógica y su entrenamiento médico contribuyeron a alcanzar tal intimidad con los mapuches de su zona, y ganar acceso a su lengua y cultura. 
Ellos se acercaban a nosotros con confianza en las diferentes necesidades de su vida, habitábamos entre ellos, los visitábamos en sus casas, curábamos a sus enfermos y compartíamos con ellos sus alegrías y tristezas. Siempre teníamos a unos indígenas por compañeros en nuestros viajes por los territorios de la Misión, y como apuntábamos cuidadosamente cada nuevo término que oíamos, venían llenándose nuestras libretas, una tras otra, con el abundante material que ahora, después del penoso trabajo de ordenarlo, entregamos a la publicidad (ix).

El Diccionario es el fruto de los primeros veinte años de trabajo de Augusta y sus colaboradores. Sin embargo, es la parte final de una trilogía de obras lingüísticas -junto con la Gramática Araucana, de 1903, y las Lecturas Araucanas, de 1910- que se conciben como un verdadero programa de aprendizaje del mapudungun. Efectivamente, es esto lo que distingue el trabajo de Augusta del realizado poco antes por Lenz (véanse sus Estudios Araucanos de 1895-1897 y su Diccionario de 1905-1910): el objetivo fundamental de Augusta es pedagógico y no meramente académico, aunque no cabe duda de que su contribución académica es inestimable. Su público explícito son los misioneros que se preparan a llevar el mensaje cristiano a los mapuches y a ayudar su progreso en general a través de la educación.

Nuestro intento ... no ha sido hacer un Diccionario histórico, sino uno del araucano moderno, que sirva a los Misioneros para poder hablar a los indígenas en un lenguaje correcto, bien inteligible para ellos (iv).

La aproximación a la labor misional de los primeros capuchinos bávaros proponía incluso elementos que hoy consideraríamos de educación bilingüe. Sabemos, por ejemplo, que ya en 1900 Augusta había preparado catequismos y abecedarios en mapudungun para su uso en las escuelas capuchinas, aunque los apoderados -en primera instancia caciques muy investidos en posicionarse en la sociedad chilena- se negaron a estas prácticas, creyéndolas poco conducentes a la rápida adquisición del castellano (v. el informe del Prefecto Röttingen de 1904, citado en Arellano 2010).

El indio, casi por regla general, prefiere libros escritos en castellano, a pesar de que, al llegar a nuestros colegios, ignore la mayoría de ellos este idioma... es necesario enseñarles ante todo, las verdades de nuestra santa religión, en su propio idioma. (Augusta 1903b: vii).

Dicho esto, debemos poner las cosas en contexto: mientras los frailes capuchinos mostraban cierto respeto por los elementos externos de la cultura mapuche, al mismo tiempo se alineaban claramente con la visión progresista imperante, que abogaba por la pronta aculturación e integración 
de los indígenas (v. Bengoa 2000). Por sobre todo, los misioneros reafirmaban los artículos de su fe católica, frente a los cuales los elementos religiosos tradicionales mapuches eran, incluso para Augusta, "estúpidos y supersticiosos" (1910: 10) y los proyectos de indigenismo más radical, como el de Panguilef (v. Bengoa 2000: 388 ff.), eran "propaganda anticristiana y antichilena" (Augusta 1934: ix). En el fondo asumían que la visión de mundo occidental primaría y que la mapuche tenía los días contados.

Es fácil prever que el idioma indígena apenas se hablará en Chile de aquí a unos cien años; la interesante y heroica raza araucana está ya por desaparecer (Augusta 1903a: viii).

Lo que nuestra obra refiere acerca de sus costumbres y supersticiones, dentro de poco ya no corresponderá a la realidad, ni se guardará memoria de ello; por eso hemos querido conservar estos detalles para la ciencia etnológica (Augusta 1910: vii).

Sin la preocupación de acelerar estos procesos, el objetivo era educar con cualquier herramienta a la mano, entre las que el mapudungun era la más fina. En tanto, Augusta tiende a abundar en ejemplificaciones lingüísticas. Ya en la Gramática se alternan lecciones, ejemplos y ejercicios, siguiendo los métodos de la época para el aprendizaje de segundas lenguas o del griego y latín. Por supuesto, el objetivo principal de las Lecturas Araucanas es dar ejemplos del uso de la lengua mapuche, aunque su valor etnohistórico es también inestimable. Los textos, provenientes de fuentes en Huapi y Panguipulli (con la cooperación de Fray Sigfrido de Frauenhäusl), presentan una sección transversal de la vida mapuche de fin de siglo XIX y principio del XX, de los cuales se extrae mucho del repertorio léxico del Diccionario, como veremos.

\section{EL DICCIONARIO: COMPOSICIÓN, ALCANCE Y ESTRUCTURA}

Sortear la brecha entre idiomas tan distintos como el mapudungun y el español trae consigo un agudizamiento de las dificultades intrínsecas del trabajo lexicográfico bilingüe. Como hace notar Catrileo (1988: 31), existe una disparidad clara en los niveles de estandarización de las lenguas involucradas, lo cual dificulta la selección de equivalencias mapuches. Esto, ya que la variabilidad geográfica, generacional y pragmática del mapudungun se encuentra escasamente descrita hoy -mucho menos un siglo atrás. Además, las profundas diferencias culturales entre la sociedad mapuche y chilena de 
principio de siglo XX traen consigo la dificultad de proporcionar mayores matices semánticos a las equivalencias léxicas, las que son fundamentalmente denotativas, mientras la constelación de posibles connotaciones permanecen bajo el sesgo del observador.

A los temas de estandarización y distancia cultual se agregan las diferencias tipológicas, entre las cuales la morfología es particularmente relevante. Mientras el español es una lengua moderadamente sintética y fusionante, el mapudungun puede ser catalogado como polisintético y aglutinante en su morfología verbal (v. Baker 2006; Zúñiga 2006). En teoría, una lengua de este tipo debiera poder reducirse simplemente a partes constituyentes únicas, con una combinatoria simple y transparente. Aunque esto es a menudo el caso, y la regularidad de la morfología -tanto en forma como en semántica- es insólita para quien hable una lengua romance, los detalles no son tan simples, cosa de la cual estaba sin duda al tanto el meticuloso Fray Félix.

Existen en realidad otros muchos vocablos araucanos, verbos-raíces y compuestos, y acepciones de palabras que no conocemos, ni hemos podido reducir todos los términos derivados a su expresión más simple (iii).

No solo es esta dificultad de descomponer los elementos morfológicos la que presenta desafíos enormes, sino también las exponenciales posibilidades combinatorias que permite la lengua, tanto a través de palabras compuestas (v. Baker y Fasola 2009; Zúñiga 2014), como de serialización verbal (v. Fernández-Garay y Malvestitti 2008) e incorporación nominal (v. Baker et al. 2005; Baker 2009). Aunque el Diccionario presenta numerosos ejemplos de palabras compuestas y derivaciones, sus elementos constitutivos no se explicitan con una glosa literal, siendo poco transparentes para el usuario no nativo.

\subsection{COMPOSICIÓN}

A pesar de que la aproximación de Augusta a temas como la morfología se encuentra empantanada entre la influencia de Bello y de la gramática escolástica (ambas miradas son muy inadecuadas al perfil tipológico del mapudungun, v. Salas 1985), su Diccionario se muestra como una obra verdaderamente moderna de lingüística descriptiva. El trabajo de campo se realiza mediante cuidadosa elicitación controlada de informantes nativos. Entre estos últimos hay tres que contribuyeron de manera más sustancial y 
cuyo rol y antecedentes son dados a conocer explícitamente, con evidente gratitud y camaradería (v). Los dos primeros, Pascual Segundo Painemilla Ñamcucheu y José Francisco Coluñ, son nativos de la zona de Budi, mientras que Domingo Segundo Huenuñamco pertenece a las cercanías de Panguipulli. Aparte de Painemilla, los otros dos informantes tienen un domino precario del español. Sin embargo, Huenuñamco habría aprendido a leer y escribir el mapudungun y mantuvo correspondencia con Fray Félix.

Augusta es muy claro en evitar confusión en la variación diatópica de las formas. Ve con preocupación la tendencia de los vocabularios antiguos a incluir cuanto tuvieran a la mano, criticando a Febrés "que sin distinción alguna ha recopilado en su libro los términos coleccionados por sus cohermanos en lugares lejanos entre sí" (vi). Así, a pesar de que Augusta tenía acceso a más material, permitió tan solo la inclusión de los elementos que él mismo había oído usar. Este principio fue su 'navaja de Ockham', tanto para la vigencia de los términos, como para su localización geográfica. En tanto, los informantes pueden tenerse como representantes de dos dialectos: el mapudungun central de Cautín (llamado "moluche" por Lenz) $\mathrm{y}$ el pehuenche hablado en Panguipulli ${ }^{4}$. Formas de la primera variedad se señalan en las entradas del diccionario con '*', las segundas con ' + '. Las formas comunes a ambas zonas no llevan marca. Mientras los vocabularios describen primordialmente variedades meridionales de la lengua -hoy deterioradas o extintas- las variedades que representa Augusta $^{5}$-en especial la central- son predecesoras de las formas de la lengua mejor descritas y preservadas hoy. Esta continuidad en el registro permite al lingüista histórico una mirada más directa al desarrollo de estas variedades de la lengua.

Parte del trabajo de elicitación constó de una revisión sistemática de los vocabularios antiguos, y el registro solo de los vocablos que eran conocidos de los informantes: "podemos garantizar que las palabras que figuran en nuestra obra las hemos oído emplear por los indígenas" (iv). Se revisaron también, cual trabajo moderno de lingüística de corpus, las obras ya disponibles en mapudungun -en particular los Estudios Araucanos de Len $^{6}$ - y se realizaron nuevas traducciones de textos religiosos, además

et al. 2015 .

5 Según Adalberto Salas, la selección de dialectos representadas por las obras de Augusta pertenecen a lo que él llama un mapuche "general" (1985: 214).

6 Aunque los objetivos para con el mapudungún de estos dos alemanes radicados en Chile difieren importantemente -el uno interesado en el aspecto práctico y pedagógico, mientras el otro, por sobre todo en lo teórico y descriptivo- tenían un gran respeto mutuo. Mientras que Augusta dice que los Estudios Araucanos por un tiempo fueron su "lectura preferida" 
de incluir lexemas extraídos de las Lecturas Araucanas, para lo cual los mismos intérpretes ayudaron en la compilación y traducción. Para el Tomo II: Español-Araucano se tomó como punto de partida la versión corriente del diccionario de la Real Academia Española, pero se elicitaron solo palabras relevantes para el contexto mapuche o misional, omitiéndose muchas palabras "por ser simplemente intraducibles, o por no tener importancia alguna para nuestro objeto" (viii).

Claramente, los tres informantes principales trabajaron con Augusta en condiciones controladas de elicitación: "les preguntábamos cien veces, hasta fastidiarlos, por dar con el sentido, la pronunciación de una palabra, el régimen de algún verbo" (iv). Sin embargo, la interacción menos estructurada del día a día y los viajes por las comunidades también fueron fuente de abundantes entradas del Diccionario. Con todo esto, aún hay un claro sesgo de Augusta por el lenguaje de registro más elevado, aclarándose que "no todo indígena es apto para servir de consultor en la indagación de su idioma, sino solamente aquellos que lo hablan con reconocida corrección" (vii).

\subsection{Contenido de las EnTRadas}

El Tomo I: Araucano-Español contiene 5.126 entradas. Éstas se organizan en orden alfabético modificado ${ }^{7}$ de acuerdo, por lo general, al morfema radical que da inicio a uno o varios lexemas -todos ellos contenidos en la entrada. En otras palabras, si existe un solo lexema correspondiente a la raíz, esta ocupa la posición de lema en la entrada (1), pero si existen varios lexemas que comienzan con la raíz (sean palabras derivadas o compuestas), uno de estos ocupa la posición de lema -normalmente un lexema independiente-y los otros se incluyen separados por ' $\|$ ' (2). Las distintas acepciones de un mismo lexema se separan con 'l' (5).

(viii), Lenz se refiere a la Gramática Araucana como "la mejor gramática ... de las lenguas indígenas americanas"(1905-1910: 923-4). Sobre la relación de los dos germanos, v. Salas (1985).

7 Los grafemas y su orden en el Diccionario son $<\mathrm{a}$, ch, d, e, ə, f, $\mathrm{y}, \mathrm{i}, \mathrm{k}, 1(1 \cdot), 11, \mathrm{~m}$, $\mathrm{n}(\mathrm{n} \cdot), \tilde{\mathrm{n}}, \mathrm{o}, \mathrm{p}, \mathrm{q}, \mathrm{r}, \mathrm{s}, \int, \mathrm{t}(\mathrm{t} \cdot), \mathrm{tr}, \mathrm{u}, \mathrm{w}, \ddot{\mathrm{u}}, \mathrm{y}>$, siendo sus valores fónicos muy a grosso modo equivalentes a los del castellano a excepción de: $\langle\mathrm{d}\rangle=[\theta],<\mathrm{ll}>=[\Lambda],\langle\mathrm{q}\rangle=[\mathrm{u}]$ y $\langle\operatorname{tr}\rangle=[\mathrm{ts}]$. Las variantes grafémicas con punto elevado son interdentales [1] $]$, [n] y [t] $]$, mientras que $<2>$, $<\mathrm{n}>\mathrm{y}<\beta>$ equivalen a AFI, [ə], [n] y [J]. Por último, $<\ddot{\mathrm{u}}>$ equivale a una vocal alta, central (o posterior), no-redondeada [i], que tiende a alternar con [ə]. Más detalles de la fonética del mapudungun central contemporáneo se encuentran en Sadowsky, et al. (2013). 
(1) yawin tr., cuidar (la siembra contra los pájaros).

(2) folil | s., la raíz. - mamoll la raíz del árbol. $\|$-entun, tr., sacar de raíz, extirpar.\|-n, n., prender (raíces). $\|-t u n, \mathrm{n}$., sacar las raíces, destroncar, n. I-unkalen, n., estar arraigado.

Como en todo el trabajo de Augusta, las consideraciones teóricas se subordinan a las prácticas, y a menudo las entradas se separan de manera más idiosincrática, de acuerdo a criterios de semántica o frecuencia. Así, encontramos dos entradas con fücha en la posición de lema; una comprende solamente el sustantivo correspondiente a 'viejo, anciano', el otro a todas las acepciones relacionadas con 'grande, largo', además de verbalizaciones con el sentido de 'viejo'. Hay seis entradas distintas que empiezan con el morfema kudaw, con acepciones relacionadas con 'trabajo', sus derivados y compuestos. En otro caso, se separan las entradas del lema pichi con sentidos relacionados a 'pequeño' de la entrada encabezada por pichike, siendo ésta una forma muy frecuente que incluye el sufijo plural propio de los adjetivos (-ke), conservando el sentido original (v.gr., 'pequeños'). En otros casos, la separación de las palabras en distintas entradas parece ser aún más arbitraria como en (3) vs. (4).

(3) filla $\mid$ s., escasez de víveres, v. g. -küyen · los meses de escasez. $\|-\eta e n$, impers., haber escasez de víveres.

(4) filla| $\boldsymbol{n}$ n., sufrir escasez.

Para el Tomo II: Español-Araucano, hay 6.991 entradas, organizadas de manera muy similar a las del Tomo I, con la excepción de que a menudo precede al primer morfema radical un prefijo derivacional, en base al cual se organizan alfabéticamente las entradas.

(5) calm|a, f., (completa de viento) üdwe. | Haber - a üdwelen, ñükun* (impers. y n.). | Estar apacible el tiempo llakolen (n. e impers.). | Le ha vuelto la -a Rupañmatui ñi illkun, ñi wefa rakiduam, kümeduamkaletui. \|l -ar, a., llakoln (tr.); (a los ánimos) llakoduameln (tr.) (Acusativo son las personas)...

Como se verá en los ejemplos (6-8), las entradas de ambos tomos incluyen una serie de lexemas, cada uno seguido de una categoría gramatical ${ }^{8}$ y su

\footnotetext{
8 Aquí: $\mathrm{s} .=$ sustantivo, adj.=adjetivo, $\operatorname{tr} .=$ verbo transitivo, $\mathrm{n} .=\mathrm{verbo}$ neutro (intransitivo),
} imper.=verbo impersonal, $\mathrm{a} .=$ verbo activo $\mathrm{f} .=$ femenino, $\mathrm{m}=$ masculino. 
equivalencia en el idioma opuesto. A menudo también se da una ilustración de la palabra en contexto.

(6) rekenkü| $\mid \boldsymbol{n}^{*}$, n., hincharse; v. g.:-kei ñi piuke Se hincha mi estómago.

(7) reke|len, n., ser como; v. g.: Eimi toro-leimi mi waponen Tú eres como un toro en tu guapeza.

(8) diversidad| f., ñi kakeumen; v. g. la - de las lenguas ñi kakeumen kewan.

A pesar de que la gran mayoría de las entradas del Diccionario incluyen como encabezado un lexema, también ocupan dicha posición unas cuarenta 'partículas', esto es, sufijos, más que nada verbales. Así vemos que el sufijo adverbial -rume (v. Salas 1992: 13; Smeets 2008: 263-4) se marca como 'partic.[ula] interp.[uesta]', con definición propia y ejemplificación (9). En algunos casos estas entradas son muy largas (v. -lle), mientras en otros Augusta refiere directamente a la Gramática (Gr. A.), en lugar de dar sentido o ilustración alguna (10).

(9) rume partic. interp., de repente; v. g.: Ñamrumei Desapareció de repente. Pẹerumei Se vió de repente.

(10) ñma partic. interp.: V. Gr. A., págs. 61-65, 298-299.

Otro aspecto en que Augusta refiere al lector directamente a la Gramática, es en la relación entre la ortografía y fonología del mapudungun. Aunque no es éste el lugar para una crítica detenida del grafemario de Augusta y su utilidad para comprender la fonología (y fonética) mapuche de un siglo atrás, cabe mencionar dos áreas donde lo escrupuloso del trabajo de Augusta genera problemas para el uso práctico de su Diccionario. Uno de estos dice relación con la llamada 'sexta vocal' del mapudungun, cuyo valor fonético varía (hoy) entre una vocal alta, central (o posterior), no-redondeada [i] y una vocal media, central, no-redondeada [ə] (v. Sadowsky et al. 2013). Tanto por encontrarse bajo la influencia de Lenz y su cuidadosa transcripción fonética, como por lo extemporáneo de un análisis propiamente fonémico, Augusta

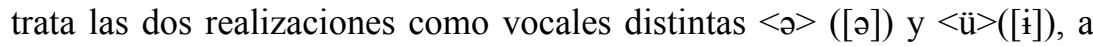
pesar de dejar muy en claro que a menudo se intercambian. El resultado es la imposibilidad de saber si un lexema con /ì/, se encontrará en el Tomo I bajo un grafema u otro. En muchos casos la entrada se duplica (v. faré = füré).

La segunda área ortográfico-fonológica que causa problemas al Diccionario es la marcación del acento, que no es consistente. Si bien, en principio, se siguen las reglas establecidas en la Gramática (1903a: 2-4), los acentos marcados a menudo corresponden a la Regla General (acentuación de la última vocal preconsonántica), donde debieran ser omitidos por 
redundancia (v. Augusta 1910: xi): es el caso de deumán 'hacer, concluir, construir, fabricar, realizar' (vs chiman 'enfermar de bubas'). Esto trae consigo la pregunta por la posición del acento en palabras que no llevan marca y cuya estructura morfológica podría interceder en su posición. De todos modos, esta inconsistencia no es rara en estudios del mapudungun, ya que tiende a haber un grado de variación en la posición del acento mapuche (v. Catrileo 2010; Molineaux 2016).

\subsection{TIPOS DE ENTRADA}

En cuanto a la cobertura léxica de la obra, no sería sorprendente que las doctrinas religiosas fuesen el objetivo principal de Augusta, dada su labor misional. Sin embargo, el interés relativamente amplio de los capuchinos lleva a un registro más variado y valioso:

en el trato con los indígenas y más especialmente con nuestros intérpretes, hemos preguntado por cuánto hay en la naturaleza que los circunda y en sus casas y costumbres, y hasta hemos abordado cuestiones de ciencias humanas (iv).

Así, mientras hay términos que son sin duda neologismos adecuados a las ideas y formas de la cultura religiosa entrante, como llapə́m-pallü-pé-yüm 'sacramento' (lit. 'cura-alma-EVIDENCIAL-CAUSATIVO') u otros que se adaptan al contexto eclesiástico, como wañó-moye-tu- $n$ 'resurrección' (lit. 'otra.vezvivir-REPETITIVO-SUSTANTIVO'), también existen gran cantidad de términos que son específicos de la cultura y modo de vida mapuche de la época, los cuales son explicados más latamente, como dəyu-machi-fe 'el hombre que anima a la machi con alocuciones, p.e., en el rewetun' (lit. 'hablar-machiAGENTE') o troytroy 'especie de bolsa con hilo para colgar, hecha de ubre de vaca u oveja y que sirve para guardar sal o ají' (un caso de reduplicación inherente, v. Zúñiga y Díaz-Fernández 2014). En algunos casos, Augusta hace notar que sus informantes perciben ciertos términos como anticuados (marcándolos 'ant.'), como en el caso de troki-we 'un hilo con que se miden los terrenos, las tareas para los segadores o de que se sirven las tejedoras para dar al telar el tamaño que desean' (lit. 'juzgar-INSTRUMENTAL') o küya (üll) 'apellido o linaje'.

A pesar de que preserva unos 50 términos 'anticuados', el grueso del Diccionario trata de mantenerse estrictamente sincrónico. Nos dice Augusta que "intencionalmente no hemos reproducido de los autores antiguos sino los vocablos que están todavía en uso hoy día o que, por lo menos, eran 
conocidos de nuestros intérpretes araucanos"(iv). Dicho afán por la lengua tal cual se hablaba en su momento, lleva también a que Augusta incluya libremente préstamos léxicos del español, no solo entre los sustantivos (akucha 'aguja', kulpan 'culpa', timun 'arado/timón'), sino también entre los adjetivos (fisaro 'perito/baqueno' [de 'bizarro'], fofo/pofo 'bobo', motro 'mocho'), y verbos (tesan 'tesar', Jofan 'sobar', kanfan 'cansarse'). Algunos préstamos son claramente más antiguos, como kawell 'caballo' y lichi 'leche', siendo capturados incluso en el vocabulario de Valdivia (1606). Los préstamos presuntamente más nuevos, como microscopio, partido, válvula o paciencia, tienden a darse con una equivalencia simple ('ídem.'), lo cual probablemente representa el hecho de que aún no tienen una adaptación estable en la fonología mapuche.

El interés profesional de Augusta sin duda da un sesgo científico a la obra, con especial énfasis en los términos médicos. Así, se incluyen, por ejemplo, añken 'cicatirz', pelleñ 'verruga', rulme-we 'esófago' (lit. tragarINSTRUMENTAL) o koñü-we 'vagina' (lit. 'parir-INSTRUMENTAL'). En algunos casos la capacidad compositiva del mapudungun sale al rescate de términos técnicos como troi-kug-foro 'radial' (hueso de la muñeca - lit. 'ramificaciónmano-hueso') o mullfuñ-kug 'pulso (de la mano)' (lit. 'sangre-mano'). Dada la naturaleza biológica de estos temas, Augusta hace notar que algunas palabras son 'muy indecentes' (marcadas con $\S \S)$ como mün 'fornincar', pifu 'membrum virile' o lludkün 'abortar'. Por último, también se incluyen otras palabras simplemente 'groseras, ordinarias' (marcadas con §), como perkü-ñma-w- $n$ 'tirarse pedos el uno al otro' (lit. 'peer-REFLEXIVO-RECIPROCOINDICATIVO-1s), kaichun 'tener diarrea'.

Un cierto interés naturalista también atraviesa el trabajo de fray Félix, incluyendo términos referentes a animales y plantas. Entre los zoonimos

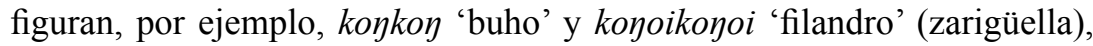
ambas reduplicaciones inherentes. Entre los fitónimos, una parte significativa de las entradas son el resultado de consultas con el entonces profesor de Botánica del Instituto Pedagógico de la Universidad de Chile, el célebre Dr. Federico Johow, quien revisó especímenes enviados por Augusta, ayudándolo a catalogar y dar nombres taxonómicos a unas 130 especies. Entre ellas se encuentran kewan'-narki 'cierta planta trepadora cuyas hojas son muy pegajosas ... Loasa gayana' (lit. 'lengua-gato') o tapitapi 'yerba San Juan ... Oenothera stricta'. 


\section{EL DICCIONARIO HOY}

Más allá de las alabanzas que recibiera Augusta de contemporáneos como Lenz, su trilogía araucana siguió siendo considerada, durante gran parte del siglo XX, "la mejor visión de conjunto de la lengua mapuche contemporánea" (Salas 1985: 215). Los últimos treinta años, sin embargo, han cambiado este panorama, con una prolifereación de trabajos lingüísticos sobre el mapudungun que permiten una visión más completa en los planos fonológico, morfosintáctico y discursivo, tomando perspectivas desde la dialectología, a la lingüística antropológica y desde la sociolingüística, a la teoría gramatical y la tipología. Sin embargo, el plano lexicográfico no ha visto el mismo desarrollo, sobre todo en cuanto respecta a cobertura (aunque haya énfasis nuevos, como el etnográfico y fonémico de Catrileo 1995).

\subsection{LIMITACIONES}

Al presentar la necesidad de su nuevo Diccionario, Augusta pone énfasis sobre cuán poco ajustados se encuentran los 'vocabularios' antiguos a la realidad mapuche de 1916:

... pero como las costumbres de los indios y todo su mundo de ideas han cambiado enteramente por el contacto con la gente civilizada y las nuevas condiciones de vida en que se encuentran, muchas de las explicaciones que dan aquellos Vocabularios, no eran ya inteligibles al intérprete ni lo son a los indios modernos en general (vii).

El mundo cultural y lingüístico mapuche ha cambiado tanto o más radicalmente en los últimos cien años que lo que habría cambiado entre la época de Augusta y los primeros vocabularios. Tras la radicación en reducciones vino un accidentado proceso de integración de los mapuche a la sociedad nacional. Tanto la industrialización como el crecimiento poblacional llevó a la necesidad económica de migración, con el resultado de que ya en los años 20 el mapuche puede ser visto como integrante de la clase obrera nacional (Bengoa 2000: 398). Particularmente importante es la incorporación mapuche al servicio militar y a la escolarización primaria masiva, que, siendo estrictamente monolingües, llevaron a la pérdida de marcadores culturales y lingüísticos. Así, de una situación de monolingüismo mapudungun con excepcional bilingüismo a principio de siglo $\mathrm{XX}$, hoy, gran parte de las comunidades mapuche han transicionado a un menguante bilingüismo, que cede espacios al monolingüismo español (Zúñiga 2007). En términos 
estructurales, la lengua misma "acusa en todos sus niveles, los efectos del contacto sistemático y permanente con el castellano" (Salas 1992: 40).

Dada la nueva realidad de forzosa convergencia cultural y lingüística del mapuche hacia la sociedad hispanoparlante, el último siglo ha visto un proceso de empobrecimiento léxico en el mapudungun. Esto no solo significa la incorporación de elementos hispanos para suplir elementos culturales foráneos a la tradición mapuche, sino también la caída en desuso de elementos nativos. Como enfatizan Chiodi y Loncón (1999), este 'depauperamiento' del mapudungun es el resultado de factores extralingüísticos, en particular, la exclusión social de sus hablantes. La superación de esta encrucijada claramente depende de factores extralingüísticos, pero requiere también un "urdir de nuevo la trama lexical de la lengua, neutralizando los efectos del olvido y de la aculturación lingüística" (ibíd: 49).

Aunque sin lugar a dudas el Diccionario nos provee de muchos de los elementos olvidados a recuperar, éste no contiene los elementos léxicos par tender puentes entre el mapudungun decimonónico y los nuevos contextos en que se encuentran inmersos los mapuches hoy. Dicha falta de actualización léxica se hace más aguda hoy en contextos urbanos (CEP 2006; Gundermann et al. 2009; Lagos 2012) en los cuales se desenvuelve la gran mayoría de quienes se autoidentifican como mapuches en Chile (INE 2003). Aunque el Diccionario puede ayudar con el afinamiento lexical del mapudungun, proveyendo las fuentes morfosintácticas y semánticas para formar neologismos, estas nuevas creaciones caen al vacío en tanto no exista un proceso de elaboración lingüística que amplíe el repertorio estilístico de la lengua. De acuerdo con Chodi y Loncón, este proceso implicaría el "despliegue de modalidades expresivas para funciones y ámbitos no tradicionales: el discurso literario, científico, tecnológico, burocrático, etc." (1999: 52).

Más allá de las trabas que los profundos cambios sociales presentan para la utilidad del Diccionario, aun permanecen los mismos desafíos propios de la tarea lexicográfica bilingüe y los problemas específicos del volumen en cuestión. En cierta medida, los problemas que esbozamos con respecto a la delimitación de las palabras y los morfemas se agudizan con el tiempo, ya que no corresponden al análisis de las gramáticas contemporáneas (Salas 1992; Zúñiga 2006; Smeets 2008) ${ }^{9}$. Lo mismo sucede con la ortografía usada

9 Véase por ejemplo el uso del término ‘acusativo’ en (5), donde el análisis contemporáneo del sufijo - el es como aplicativo o específicamente de benefactivo (v. Zúñiga 2010; Golluscio 2010), más que de objeto directo, cómo pareciera entenderlo aquí Augusta. 
por Augusta, que antedata a la teoría fonémica, y que contrasta con los al menos cinco grafemarios en uso hoy (v. Zúñiga 2001).

\subsection{ReLEVANCIA}

A pesar de la marginalización y concomitante desprestigio de los mapuches y su lengua durante el siglo pasado, estudios recientes como los de Gundermann et al. (2009) y Lagos (2012) muestran una revaloración positiva del mapudungun como fuente de identidad mapuche. Es bajo este contexto que, tras al menos cien años de pérdida léxica, quizás sea razonable creer que la obra de Augusta ayude a inclinar la balanza a favor del mapudungun. Lo crucial a notar aquí es que un diccionario tiene un fuerte valor simbólico, en tanto es receptáculo de las unidades intuitivamente básicas de la lengua: sus palabras. Como tal, constituye un elemento importantísimo dentro del corpus existente del mapudungun, que a su vez es condición para el desarrollo de los procesos de afinamiento léxico y elaboración lingüística (v. Chiodi y Loncón 1999).

En la particular situación de desventaja en que se encuentra el mapudungun frente al español, la revitalización de la lengua es bastante improbable sin algunos elementos de planificación, sean ellos explícitos o implícitos (v. Lagos y Espinoza 2013). En este contexto, independiente de la plataforma socio-cultural, política e institucional que permita dar pasos en esta dirección, vemos que el diccionario de Augusta provee abundantes modelos de ejemplaridad para el uso de la lengua, dando directrices para su elaboración (v. Gallardo 1984).

Los mecanismos para extender el repertorio léxico del mapudungun son, en efecto, procesos que se efectúan en todas las lenguas y que, para el mapudungun, se encuentran bien documentadas en el Diccionario: la derivación, la composición y la extensión semántica, además del uso de préstamos léxicos. Hoy, entre los neologismos mapuche compiten muy de cerca la formación mediante recursos propios de la lengua y los préstamos léxicos del español. A pesar de la innegable influencia del español, los recursos internos del mapudungun muestran una versatilidad enorme, permitiendo gran creatividad léxica en su uso (v. Villena 2014 para el caso de hablantes en Santiago). El diccionario de Augusta proporciona importantes modelos para esta labor creativa. 
Cuadro 1: Paralelos entre el Diccionario de Augusta y la creación de neologismos espontáneos en Villena (2014) 'V’y Chiodi y Loncón (1999) 'CL ${ }^{10}$

\begin{tabular}{|c|c|c|}
\hline & Augusta (1916) & Neologismos espontáneos \\
\hline Derivación & $\begin{array}{l}\text { rekül-tu-we } \\
\text { arrimar-VERB-INSTR } \\
\text { 'respaldo' }\end{array}$ & $\begin{array}{l}\text { pelom-tu-we }(\mathrm{V}) \\
\text { luz-VERB-INSTR } \\
\text { 'ampolleta' }\end{array}$ \\
\hline Derivación & $\begin{array}{l}\text { kaiñe-wen } \\
\text { enemigo-RECIP } \\
\text { 'enemigos entre sí' }\end{array}$ & $\begin{array}{l}\text { kudaw-fe-wen (CL) } \\
\text { trabajo-AGENTE-RECIP } \\
\text { 'compañeros de trabajo' }\end{array}$ \\
\hline Composición & $\begin{array}{l}\text { nie-kütran-we-ruka } \\
\text { tener-enfermendad-INSTR-casa } \\
\text { 'hospital' }\end{array}$ & $\begin{array}{l}\text { lawen-ruka } \quad(\mathrm{V}) \\
\text { remedio-casa } \\
\text { 'farmacia' }\end{array}$ \\
\hline Composición & $\begin{array}{l}\text { nor-dəyun } \\
\text { recto-hablar } \\
\text { 'hablar sinceramente' }\end{array}$ & $\begin{array}{l}\text { aye-kan-düngun (CL) } \\
\text { reír-CONT-hablar } \\
\text { 'contar chistes' }\end{array}$ \\
\hline Extensión Semántica & $\begin{array}{l}\text { wigka-pülku } \\
\text { extranjero-chicha } \\
\text { 'aguardiente' }\end{array}$ & $\begin{array}{l}\text { wingka-kareta }(\mathrm{V}) \\
\text { extranjero-conT-carreta } \\
\text { 'automovil' }\end{array}$ \\
\hline Extensión Semántica & $\begin{array}{l}\text { yawilma* } \\
\text { choroy } \\
\text { 'gente locuaz' }\end{array}$ & $\begin{array}{l}\text { ñua-ka-fe (CL) } \\
\text { engañar-CONT-AGENTE } \\
\text { 'actor' }\end{array}$ \\
\hline
\end{tabular}

Otro aspecto en el que el Diccionario es sin duda de gran valor es en la exploración diacrónica del mapudungun, presentando un punto de fuga robustamente atestiguado dentro de los 400 años de la historia documentada de la lengua. A pesar de que la lingüística histórica de una lengua americana pareciera ser un área excesivamente disociada de los problemas más urgentes y prácticos de una lengua amenazada, los datos lingüísticos son fundamentales en la reconstrucción de relaciones culturales y movimientos poblacionales para los cuales no hay registro narrado y los datos arqueológico/genéticos son de difícil interpretación. De una manera u otra, las raíces lingüísticas influyen también en temas identitarios, que a su vez influyen en la vitalidad lingüística.

10 Se usan las siguiente abreviaturas: $\mathrm{VERB}=$ verbalizador; INSTR $=$ instrumental; $\mathrm{RECIP}=$ marcador recíproco; AGENTE $=$ agentivizador; $\mathrm{CONT}=$ continuativo. 
A pesar de que, como hemos visto, el trabajo de Augusta es estrictamente sincrónico, este mismo hecho nos permite una vista de las palabras que han caído en desuso en relación con los vocabularios -al menos en las áreas donde fray Félix realizó su trabajo de campo. Dicho esto, las formas del diccionario no pueden representar solo el principio del siglo XX, sino también capas más profundas a través de los elementos gramaticalizados y lexicalizados (v. Payne 1984; Zúñiga pendiente). Nos muestra, además, cómo los mapuches de hace más de cien años habrían oído y reproducido los elementos del sistema fonológico del español de Chile y Argentina en tanto lo incorporaban al propio a través de prestamos (v. ofida/ofisa* $/$ ofifa ${ }^{+}$ 'oveja', manfun. ${ }^{+} / \operatorname{man} \int u n^{*}$ 'buey (manso)') ${ }^{11}$.

Al igual que las Lecturas Araucanas, el Diccionario continúa teniendo un inmenso valor etnográfico, dejando entrever las diferencias culturales específicamente mapuches de la época. El foco de Augusta no es sobre la derivación en castellano, por ejemplo, sino en mapudungun: ruka-fe es 'quien sabe hacer casas' no 'arquitecto' (Salas 1985; Gallardo 1986). De este modo, se dejan entrever muchos otros aspectos de la cultura, como la asignación de valor, la construcción del entramado social (véanse las relaciones de parentesco) y la relación con el mundo natural y espiritual.

\subsection{Prospectos}

El Diccionario de Augusta, en su grandeza, proyecta una larga sombra. Frente a la riqueza léxica que nos muestra -con el trasfondo de la cultura tradicional mapuche-, es difícil no sentir que el estado actual del la lengua es oscuro. Esta visión nostálgica, sin embargo, no es exclusiva del mapudungun. Las lenguas cambian al hablarse, de lo contrario internalizan su supuesta inadecuación, se anquilosan y mueren. Sin lugar a dudas hay escollos aparentemente infranqueables en el camino a la plena revitalización lingüística, pero existe una batería de provisiones, entre las cuales el Diccionario de Augusta no debe ser subestimado.

Las posibilidades del diccionario aún son muchas. En 1916, Augusta planteaba: "Muy interesante sería el trabajo que propone el Dr. don Rodolfo Lenz, de hacer un Diccionario colectivo de las palabras contenidas tanto en

11 Esta es un área interesantísima que han explorado Hasler y Soto (2012) para ciertos aspectos de las consonantes chilenas en contraste con los vocabularios mapuche de los siglos XVI y XVII. 
los Vocabularios antiguos, como en este nuevo, transcribiendo aquellas a la fonética moderna" (iv). Aunque entonces rechazara esta idea en pos del rol pedagógico y misional de su trilogía de obras, este trabajo está pendiente aún. Un excelente principio de esta labor se ha dado en el análisis detallado de las palabras de origen mapuche en el DRAE del profesor Gilberto Sánchez (2010). Dicho trabajo examina 302 entradas del diccionario español y propone brindarles el nombre de mapuchismos, dando cuidadosa cuenta de las formas atestiguadas en las obras lexicográficas coloniales y modernas (tanto del mapudungun como del español) y examinando sus primeros usos en obras en castellano colonial.

La extensión de un trabajo como el del profesor Sánchez a los elementos léxicos presentes en las obras coloniales y modernas del mapudungun -independiente de su presencia en diccionarios españoles- sería sumamente útil para establecer una primera etimología del mapudungun y cumplir el sueño compartido por Lenz y Augusta. Idealmente, un trabajo de este tipo incluiría un análisis de la fonología y morfología de estos elementos en las distintas etapas históricas. Tal tarea empieza a ser posible a través de dos proyectos en desarrollo. El primer aporte corresponde al Corpus Lexicográfico del Mapudungun (CORLEXIM - Villena et al. 2016), basado en la universidad Pampeu Fabra y que compila las 32.299 entradas de los vocabularios y diccionarios de Valdivia (1606), Febrés (1765) y Augusta (1916) y propone transcripciones fonémicas de cada una. El segundo aporte corresponde al proyecto Sounds of Mapudungun (Sadowsky et al. 2014) basado en la Universidad de La Frontera y el Departamento de Lingüística del Instituto de Antropología Evolutiva Max Planck. Este último, recoge material fonético/fonológico contemporáneo a lo largo de gran parte del área de habla mapuche, para trazar sus relaciones sincrónicas y diacrónicas, aportando así a la reconstrucción de las etapas más antiguas de la lengua y su dispersión geográfica y temporal (v. Sadowsky et al. 2015).

Para volver a la pregunta inicial: ¿Por qué no ha sido superado el Diccionario de Augusta? ¿Es por falta de material que documentar, o por falta de mejor documentación? La respuesta, como el lector atento habrá discernido, cae en medio de ambas alternativas. Los nuevos elementos léxicos del mapudungun son muy distintos de aquellos que documentaba Augusta: son menos uniformes, más especulativos y aun menos estándares, pero no por ello menos reales y nativos. Por otro lado, el desafío para quienes documentan la lengua hoy es elicitar estos usos nuevos, hacerlos más visibles y quitar el estigma de que el mapudungun es una lengua congelada en el tiempo. El diccionario de Augusta representa la tradición, pero también es un repositorio para la creatividad léxica del hablante en su interacción con 


\section{un mundo cambiante. La tarea lexicográfica del mapudungun, entonces, no} se encuentra terminada.

\section{REFERENCIAS BIBLIOGRÁFICAS}

Arellano, Carmen. 2010. "Las opiniones erróneas que ... circulan en la capital". La percepción cultural entre los wingka y los mapuche. Revista Chilena de Literatura, Sección miscelánea: 113-50.

Augusta, Félix José. 1903a. Gramática Araucana. Valdivia: Imprenta Central J. Lampert.

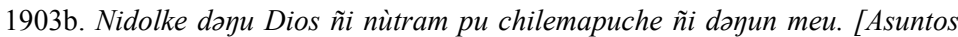
principales de historia divina en la lengua de los mapuches de Chile]. Freiburg im Breisgau: B. Herder.

1910. Lecturas Araucanas (1 ${ }^{\mathrm{a}}$ edición). Padre Las Casas: Editorial San Francisco. 1916. Diccionario Araucano-Español y Español-Araucano. Santiago: Imprenta Universitaria.

1934. Lecturas Araucanas (2a edición). Padre Las Casas: Editorial San Francisco.

Baker, Mark C. 2006. On zero agreement and polysynthesis. En Peter Ackema, Patrick Brandt, Maaike Schoorlemmer, y Fred Weerman (eds.). Arguments and agreement, pp. 289-320. Oxford: Oxford University Press.

2009. Is head movement still needed for noun incorporation? Lingua 119: 148-65.

Baker, Mark C., y Roberto Fasola. 2009. Araucanian: Mapudungun. En Rochell Lieber y Pavol Štekauer (eds.). The Oxford handbook of compounding, pp. 594-608. Oxford: Oxford University Press.

Baker, Mark C., Roberto Aranovich, Lucía A. Golluscio. 2005. Two types of syntactic noun incorporation: Noun incorporation in Mapudungun and its typological implications. Language 81 (1): 138-76.

BARBARÁ, Federico. 1879. Manual ó vocabulario de la lengua pampa. Buenos Aires: Imprenta C. Casavalle.

Bengoa, José. 2000. Historia del pueblo mapuche (Siglos XIX y XX). Santiago: Lom Ediciones.

Cañas, Alejandro. 1911. Estudios de la lengua veliche. Santiago: Imprenta, Litografía y Encuadernación "Barcelona".

Cañumil, Tulio, Darío Cañumil y Marta Berretta. 2016. Mapuchezungun-wigkazugun pici hemvlcijka / Pequeño diccionario castellano-mapuche. Buenos Aires: Impresión independiente.

Catrileo, Maria. 1995. Diccionario lingüístico-etnográfico de la lengua mapuche. Santiago: Editorial Andrés Bello.

1988. "Diseño de un diccionario lingüístico-etnográfico del mapudungun". Documentos lingüisticos y literarios 14: 31-36.

2010. La lengua mapuche en el siglo XXI. Valdivia: Facultad de Filosofía y Humanidades, Universidad Austral de Chile.

CEP (Centro de Estudios Públicos). 2006. Estudio de opinión pública: Los mapuche rurales y urbanos hoy - Documento de trabajo N³67. Santiago Centro de Estudios Públicos. http://www.cepchile.cl/estudio-de-opinion-publica-los-mapuche-rurales-y-urbanos-hoymayo-2006/cep/2016-03-04/094057.html [15 de Junio, 2016]. 
Chiodi, Francesco y Elisa Loncón. 1999. Crear nuevas palabras: Innovación y expansión de los recursos lexicales de la lengua mapuche. Temuco: Instituto de Estudios IndígenasCONADI.

Croese, Robert. 1980. Estudio dialectológico del mapuche. Estudios filológicos 15: 7-38.

Croese, Robert, Adalberto Salas y Gastón Sepúlveda. 1978. Proposición de un sistema unificado de transcripción fonémica para el mapudungun". Revista de lingüistica teórica y aplicada 16: 151-59.

Erize, Esteban. 1960. Diccionario comentado mapuche-español. Buenos Aires: Universidad Nacional del Sur.

Falkner, Thomas. 1774. A description of Patagonia. Hereford: C. Pugh.

Febrés, Andrés. 1765. Arte de la lengua general del Reyno de Chile. Lima.

FernÁndez-Garay, Ana y Marisa MalvestitTi. 2008. Construcciones verbales seriales en mapudungun. Lexis 31 (1): 33-48.

Gallardo, Andrés. 1984. La situación mapuche, problema de planificación lingüística. Cultura, hombre, sociedad (CUHSO) 1 (1): 151-88.

1986. Fray Félix José de Augusta y el Diccionario araucano". Cultura, hombre, sociedad (CUHSO) 1 (3): 173-91.

Golluscio, Lucía. 2010. Ditransitive constructions in Mapudungun. En Andrei Malchukov, Martin Haspelmath, y Bernard Comrie (eds.). Studies in ditransitive constructions: A comparative handbook, pp. 710-56. Berlin: Mouton de Gruyter.

Gundermann, Hans, Jaqueline Canihuan, Alejandro Clavería y César Faúndez. 2011. El mapuzungun, una lengua en retroceso. Atenea 5 (03): 111-31.

Gundermann, Hans, Luis Godoy, Jacqueline Caniguan, Elías Ticona, Ernesto Castillo, Alejandro Clavería y César Faúndez. 2009. Perfil sociolingüístico de comunidades mapuche y aymara en la Región Metropolitana. Santiago: Corporación Nacional de Desarrollo Indígena y Universidad Tecnológica Metropolitana.

Hasler, Felipe y Guillermo Soto. 2012. Determinación de algunas propiedades del subsistema consonántico del mapudungun y el del español de Chile en los siglos XVI y XVII a partir de los hispanismos léxicos". En Hebe A. González y Beatriz Gualdieri (eds.). Lenguas indigenas de América del Sur I: Fonología y léxico, pp. 92-102. Mendoza, Argentina: Editorial de la Facultad de Filosofía y Letras de la Universidad Nacional de Cuyo.

Havestadt, Bernhard. 1777. Chilidugú: Sieve tractatus linguce Chilensis. Leipzig: Treubner.

Herckmans, Elias. 1907. El vocabulario araucano de 1642-1643. En Rodolfo R. Schuller (ed.). Santiago: Imprenta Cervantes.

Hernández, Arturo, Nelly Rámos y Carlos Cárcamo. 1997. Lengua y cultura mapuche: Diccionario Mapudungun, Español, Inglés. Santiago: Pehuén.

INE. 2003. Síntesis de resultados: Censo 2002. Santiago: Comisión Nacional de XVII Censo de Población y Vivienda. Disponible en http://www.ine.cl/cd2002/ sintesiscensal.pdf [Consulta: 30/03/2016].

Lagos, CRISTIÁN. 2012. El mapudungun en Santiago de Chile: Vitalidad y representaciones sociales en los mapuches urbanos. Revista de lingüistica teórica y aplicada 50 (1): 161-84.

Lagos, Cristián, y Marco Espinoza. 2013. La planificación de la lengua mapuche en Chile a través de la historia. Lenguas Modernas 42: 47-66.

Lenz, Rodolfo. 1895-1897. Estudios Araucanos. Santiago: Anales de la Universidad de Chile. 1905-1910. Diccionario etimológico de las voces chilenas derivadas de lenguas indigenas americanas. Santiago: Editorial Universitaria.

MolineauX, Benjamin J. 2016. "Native and non-native perception of stress in Mapudungun: Assessing structural maintenance in the phonology of an endangered language." Language and Speech [online first]. 
Olivera, Miguel. 1966. Reseña de Esteban Erize, Diccionario comentado mapuche-español. Nueva revista de filología hispánica XVIII: 187-88.

PAYNe, DaVID L. 1984. Sobre el desarrollo histórico de los sufijos de referencia cruzada del mapudungun. Temuco: Universidad de la Frontera / Instituto Lingüístico de Verano.

Sadowsky, Scott María José Aninao, María Isabel Cayunao, y Paul Heggarty. 2015. Huilliche: ¿Geolecto del mapudungun o lengua propia? Una mirada desde la fonética y la fonología de las consonantes. En Ana Fernández Garay y María Alejandra Regúnaga (eds.). Lingüística indígena sudamericana, pp. 23-51. Buenos Aires: Facultad de Filosofía y Letras, Universidad de Buenos Aires.

Scott, Sadowsky, María José Aninao, Paul Heggarty, María Isabel Cayunao Nahuelcheo, María Ignacia Fuentes Burgos, Catalina Sandoval Muñoz, Erik Farías Castro, Margaret Mora Ortega y Jakob Runge. 2014. The 'Sounds of Mapudungun Project' [en línea]. Disponible en http://sadowsky.cl/mapu/index.html [Consulta 30/03/2016].

Scott, Sadowsky, Héctor Paniqueo, Gastón Salamanca y Heriberto Avelino. 2013. Mapudungun. Journal of the International Phonetic Association: Illustration of the IPA 43 (1): 87-96.

Salas, Adalberto. 1985. Fray Félix José de Augusta: Su aporte a los estudios de la lengua y la cultura de los mapuches o araucanos". Cultura, hombre, sociedad (CUHSO) 2 (2): 197-272.

1992. El mapuche o araucano. Madrid: MAPFRE.

SÁnchez, Gilberto. 2010. Los mapuchismos en el DRAE. Boletín de Filología XLV (2): $149-256$.

SMeets, IneKe. 2008. A grammar of Mapuche. Berlin: Mouton de Gruyter.

VALDIVIA, LuIS DE. 1606. Arte y gramatica heneral de la lengua que corre en todo el Reyno de Chile, con un vocabulario y confessionario. Sevilla: Thomás López de Haro.

Villena, BelÉn. 2014. Creación neológica en mapudungún: Entre el desplazamiento y la lealtad lingüística. Terminàlia 10: 37-49.

Villena, Belén, Andrés Chandía, Isabel Araya y Cecilia Flores. 2016. Corpus lexicográfico del mapudungun (CORLEXIM). http://www.chandia.net/corlexim [30 de Marzo, 2016].

ZúÑIga, Fernando. 2001. Escribir en mapudungun: Una nueva propuesta. Onomazein 6: $263-79$.

2006. Mapudungun: El habla mapuche. Santiago: Centro de Estudios Públicos.

2007. Mapudunguwelaymi am? ‘AAcaso ya no hablas mapudungun?’ Acerca del estado actual de la lengua mapuche. Estudios Públicos, pp. 9-24.

2010. Benefactive and malefactive applicativization in Mapudungun. En Fernando Zúñiga y Seppo Kittilä (eds.), Benefactives and malefactives: Typological perspectives and case studies, pp. 203-18. Amsterdam: John Benjamins.

2014. Nominal compounds in Mapudungun. En Swintha Danielsen, Hatja Hannss y Fernando Zúñiga (eds.). Word formation in South American languages, pp. 11-32. Amsterdam: John Benjamins.

pendiente. An exploration of the diachrony of mapudungun valency-changing operations. En Leonid Kulikov y Seppo Kittilä (eds.). The diachronic typology of voice and valency-changing categories, Amsterdam: John Benjamins.

ZúNiga, Fernando y Antonio Díaz-Fernández. 2014. Reduplication in mapuzungun: Form and function. En Gale Goodwin y Hein van der Voort (eds.), Reduplication in indigenous languages of South America, pp. 17-38. 2. Leiden: Brill. 
